\title{
Lessons Learned and Ideas Formed from Early Studies of pA Collisions
}

\author{
L. McLerran ${ }^{\mathrm{a}, \mathrm{b}, \mathrm{c}}$ \\ ${ }^{a}$ Physics Dept, Bdg. 510A, Brookhaven National Laboratory, Upton, NY-11973, USA \\ ${ }^{b}$ RIKEN BNL Research Center, Bldg. 510A, Brookhaven National Laboratory, Upton, NY \\ 11973, USA \\ ${ }^{c}$ Physics Department, China Central Normal University, Wuhan, 430079, China
}

\begin{abstract}
I discuss ideas that were developed in the early studies of high energy proton nucleus collisions.
\end{abstract}

\section{Introduction}

In 19878 I was a postdoctoral fellow at the Stanford Linear Accelerator Center (SLAC). I became interested in heavy ion collisions and began having conversations with Miklos Gyulassy who was at that time a Fellow in the Nuclear Science Division of Lawrence Berkeley Laboratory (LBL). I was also discussing with James Bjorken who was a faculty member at SLAC. I was interested to see if ultra-relativistic heavy ion collisions might provide the environment where one could make and study novel new forms of matter, such as the Quark Gluon Plasma. To address this question one needed a space-time picture of high energy hadronic interactions motivated by what was known from experiments. At that time there were seminal ideas which were embodied in several very important papers that I read.

One of these papers was that of Wit Busza, "Review of Experimental Data on Hadron Nucleus Collisions at High Energies." [1] Wit was at that time a young professor at the Massachusetts Institute of Technology (MIT). To give you an idea of the contents of the review, I quote the abstract:

"In this review an attempt is made to summarize briefly all that is presently known experimentally about hadron-nucleus collisions at high energies. Comparisons with theoretical models are kept to a minimum. However, an outline of some theoretical ideas that have been put forward in interpreting the data is included"

Wit's style, which is largely time translational invariant, comes through very clearly in the way the article is written: An experimentalist reports experimental results, and then does a skeptical comparison with various theoretical ideas.

Another very important paper I read was due to Bjorken, "Hadron Final States in Deep Inelastic Processes"[2]. These were lecture notes given at the International Summer Institute in Theoretical Physics, in DESY in Sep. 
1975. They reflect Bjorken's deep and intuitive understanding of the underlying space-time dynamics of strong interactions.

In both of these papers, there is a strong relation to thoughts that have arisen from the Krakow school of Andrzej Bialas of the Jagiellonian University. [3] The classic wounded nucleon model arose from this school in the work of Bialas, Bleszynski and Czyz, and form a basic component of most pictures of heavy ion collisions and proton nucleus collisions[4]. The work in Poland on the wounded nucleon model which would prove essential for the development of the field, and it's initial motivation was based upon the experimental results on pA collisions of Busza and colleagues.[1]. The works of Bjorken and Busza served in part for me as an introduction to the ideas being developed in Poland, which I studied and tried to understand, and ultimately led for a long association with Bialas and his colleagues in Krakow

Bjorken's lectures taught me two very important ideas. The first is the correlation between momentum space and coordinate space which is associated with Lorentz time dilation. This is very important for production of bulk forms of matter. Bulk matter is in some volume at some time with some energy density. These are concepts wedded to coordinate space. On the other hand, in collisions one measures only the momentum space distributions of particles. Bjorken's lectures provided the bridge that allowed one to use the information gathered by measuring momentum space distributions and convert it into information about energy densities of matter produced in volumes much larger than the typical separation of produced particles. Of course one wants to make the spatial dimensions as large as possible compared to the typical particle separation, and so one is led to nuclei, but one also needs to understand the longitudinal volume in which the matter is made, and the evolution in time. This is where Bjorken's insight is essential. The second very important idea was the inside-outside cascade and its space time dynamics. This cascade describes the production of particles and ultimately leads to the picture we now all accept in heavy ion collisions of matter that is produced which is expanding in the longitudinal direction. I will explain in more detail below.

\subsection{The Correlation between Momentum and Coordinate}

A high momentum hadron may be thought about in terms of its quark and gluon constituents. The longitudinal position, $z$, of such a constituent relative to the position of a hadron is given by the uncertainty principle, $\delta z \sim 1 / p_{z}$ When soft (low transverse momentum) hadrons are produced, it takes a characteristic time, in the particles rest frame, of order $t_{0} \sim 1 / \Lambda_{Q C D} \sim 1 \mathrm{Fm}$. In the frame where the particle is moving, the time is

$$
t \sim \gamma t_{0}
$$

In this equation, the Lorentz gamma factor of the hadron is $\gamma$. The particle will therefore be produced at a longitudinal coordinate

$$
z \sim \gamma t_{0}
$$


If we express things in terms of the longitudinal rapidity $y$, we see that

$$
y=\frac{1}{2} \ln \left(\frac{t+z}{t-z}\right)=\frac{1}{2} \ln \left(\frac{1+z / t}{1-z / t}\right)=\frac{1}{2} \ln \left(\frac{1+v_{z}}{1-v_{z}}\right)=\frac{1}{2} \ln \left(\frac{E+p_{z}}{E-p_{z}}\right)
$$

In deriving this equation, I have assumed that $t$ and $z$ are large compared to any uncertainty in the place where the scattering took place where the production of the particle was initiated. This is a good approximation when $\gamma>>1$. The above equation is the essence of the inside-outside cascade description, and shows the correlation between longitudinal momentum and position.

\subsection{The Matter is Produced with Longitudinal Expansion}

A picture of longitudinally expanding matter is shown in Fig. 1. The position where the particles are produced is proportional to the particle's momentum. This is the $1+1$ dimensional analog $3+1$ dimensional Hubble expansion. The picture and the resulting expansion are clearly invariant under Lorentz boosts along the $z$ axis.

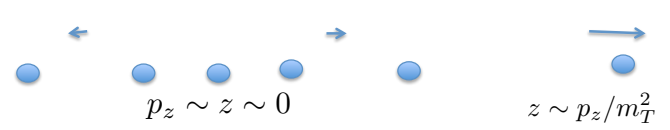

Figure 1: A pictorial representation of particle production and it's associated longitudinal expansion.

This picture is the essence of the inside-outside cascade. For example, if we imagine a pair of very high energy quarks being produced in an $e^{+} e^{-}$collision, and we look in the center of mass frame, we think of the produced hadrons as being fragments of the jets made by the high energy quark-antiquark pair. The particles first produced are low momentum particles close to the point of collision. The higher momentum particles are produced at later times farther away from the collision.

This picture leads to a simple description of the initial energy deposition in heavy ion collisions. Together with Anishetty and Koehler,[5] I applied this to the fragmentation region of heavy ion collisions, estimated the energy density, and concluded there was sufficient energy density and long enough time scales to make interesting forms of matter such as a Quark Gluon Plasma. These estimates relied upon the experimental observations discussed in Busza's review.[1] I also estimated the energy density produced in the central region of ultra relativistic heavy ion collisions, but the estimate was not particularly reliable because at that time there was not a measurement of the density of particles produced at zero rapidity in the center of mass frame for high energy collisions. All the data was for either the fragmentation region of the target or projectile. It took higher energy than was available at the time to separate these fragmentation regions and make a clean well defined central region. Bjorken, in his classic and beautiful paper about hydrodynamics provided an elegant and 
simple derivation of a formula for such ultra-relativistic collisions that redefined the problem: Measure the multiplicity and then you have determined the energy density at some time after the collision.[6] He also formulated hydrodynamic equations for the subsequent evolution of matter produced in the collision. (I was aware of the possibility to formulate a hydrodynamic description of these collisions, but I was stuck in the complications of the fragmentation region and did not think hard enough about the central region. The central region is so much simpler.)

\subsection{Early Ideas Concerning the Inside-Outside Cascade}

The ideas concerning the inside-outside cascade originated only in part from pure thought. They first appear in the Landau-Pomeranchuk-Migdal treatment of electromagnetic cascades[7]-[8]. In Fig 2, a high energy photon disassociates

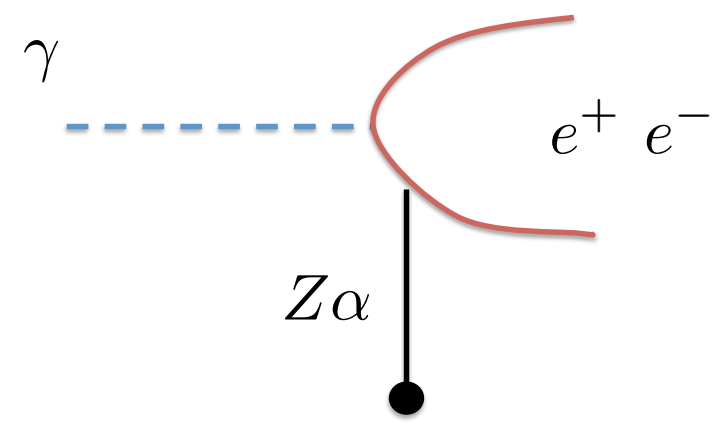

Figure 2: A graphical representation of pair production by a photon in an electromagnetic cascade.

into an electron-positron pair. This pair a made real by interacting with a nucleus of charge $Z e$. The time to form this pair in its rest frame is of order $1 / m$ where $m$ is the electron mass. This electron pair travels a distance of order $\gamma / m$ during this time, where $\gamma$ is the Lorentz gamma factor of the pair, $\gamma \sim E / m$, and $E$ is the energy of the photon. If the distance it takes for this pair to form is larger than the typical mean free path for particle formation, there will be no possibility to produce the pair. This means that the development of the electromagnetic cascade is suppressed relative to what is naively expected. The distances involved for the highest energy cosmic rays are substantial: Electronpositron pairs are produced over distance scales of tens of meters.

\section{Busza's Review}

I learned from Busza's review was

- What is known?

- What is fantasy? 
- What do we need to learn?

There were discussed a number of very important issues related to understanding high energy nucleus-nucleus collisions. These ideas remain important for today's research, although they are sometimes now formulated in different terms.

\subsection{Cross Sections}

"Total hadron-nucleus cross sections at high energies show no surprises-to within experimental and theoretical uncertainties they can be derived by hadronnucleus cross sections using the Glauber model"

This has been verified in subsequents experiments at the AGS and RHIC at BNL and the SPS and LHC at CERN. Glauber theory works very well for the description of the gross properties of nuclear collisions. The deviations from Glauber theory in the RHIC and LHC experiments in fact provide a measure of new physics.

\subsection{Coherent Production}

"If coherent production of multi-particle states is analyzed in terms of the Glauber model, one observes an apparent transparency of nuclear matter to newly produced states."

I think this was the beginning of ideas associated with color transparency. Color transparence is induced when a color neutral probe produces an overall color natural pair of colored particles. It takes time for these color states to separate, and at high energies, this time is Lorentz time dilated. If the colored pair are close together, their average summed color field results from canceling contributions, and the pair does not interact as strongly as would have been thought if the color pairs separated by a distance of order $1 \mathrm{Fm}$. . Data supporting this conclusion are shown in Fig. 3

\subsection{Lack of Intra-Nuclear Cascading}

"The most striking feature of multi-particle production in hadron-nucleus collisions is its weak A dependence. There is little if any evidence of the buildup of an intra-nuclear cascade."

Perhaps the most dramatic evidence for the Landau-Pomeranchuk-Migdal effect in hadron interactions was the lack of intra-nuclear cascading for the high energy particle produced when a high ever hadron hits a nucleus at rest. This can be used to estimate a hadron formation time. It is shown in Fig. 4 of Busza's review.

\subsection{Multiplicity of Particles Produced in pA Collisions}

"Another totally unexpected result in hadron-nucleus collisions is the A dependence of the inclusive particle production spectra at large transverse momentum."

"It is possible that the interpretation of these data is uninteresting" 


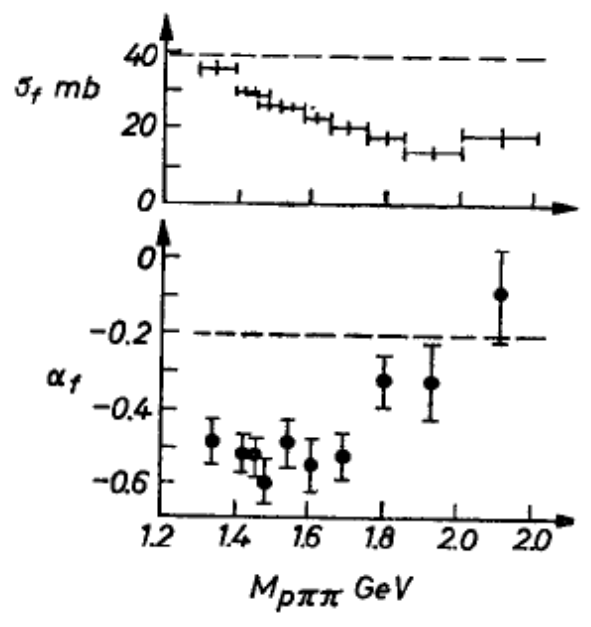

Figure 3: The example of transparency shown in Busza's review: Apparent transparency of nuclei to a coherently produced multi-hadron state. $\sigma_{f}$ is the extracted total $\left(p \pi^{+} \pi^{-}\right)$-nucleus cross section from a Glauber analysis of $p+A \rightarrow p \pi^{+} \pi^{-}+A$ data at $22.5 \mathrm{GeV}$.

The style of the last comment speaks for itself.

The issue of the dependence of the central region multiplicity was not determined at that time, nor in my opinion is it resolved yet at asymptotically high energy for arbitrarily large nuclei. See [9] for a recent article on the controversy. In Bj's summer school lectures at DESY, it was assumed that the multiplicity goes like $A^{1 / 3}$ in the nuclear fragmentation region but otherwise if of $0(1)$. In Fig. 5, Bjorken's plot from his summer school lectures is shown. Such a prediction, up to logarithms of the nuclear thickness is that of the Color Glass Condensate (CGC) description as well. (The CGC is a hypothetical form of matter associated with the high density of gluons in the initial state of hadrons in high energy collisions.) The wounded nucleon model predicts $A^{1 / 3} / 2$, and this is consistent with the data. What is difficult to disentangle is the logarithmic depndence found in the CGC and how this can imitate the wounded nucleon model.[3] Busza found his experimental results to be consistent with the wounded nucleon model.

At the time of my work with Anishetty and Koehler, we were unsure as to what to use for the central region mutlplicity in AA collisions. Because of this uncertainty, we were unable to make a reliable prediction. Bjorken turned this issue into a prediction for the central region energy density based upon an experimental measurement of the high energy multiplicity in AA collisions. Although experimentalists used Bjorken's formula to get a bound on the energy density in AA collisions at SPS energies, these energies are not high enough for this formula to work. The Lorentz contracted nuclear size in the central rapidity region of such collisions is too large. The first reliable determination was done 

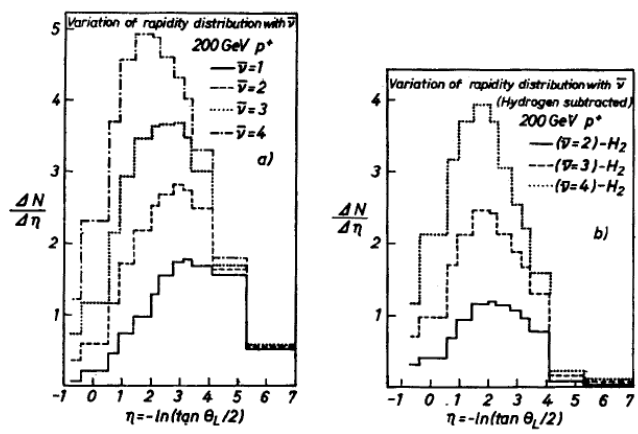

Figure 4: Example of the variation of pseudo-rapidity distributions with the size of a nuclear target. The quantity $\bar{\nu}$ is a measure of the average nuclear thickness in units of the mean free path of protons in nuclear matter. $N$ is the average number of charged particles produced in an inelastic collisions including both pions and recoil protons.

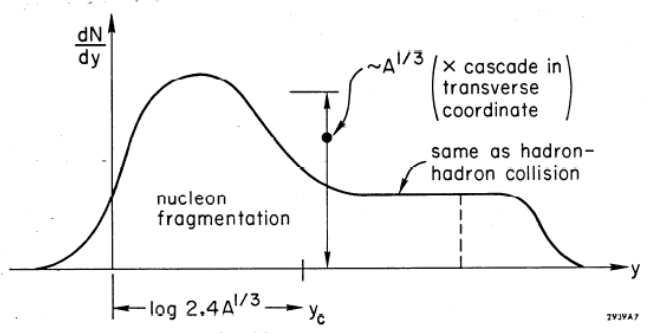

Figure 5: The multiuplicity of produced particles in a pA collision as a function of rapidity from Bjorken's lectures.

at RHIC, in the PHOBOS Experiment. Busza was the spokesperson.[10] This was the first reported experimental result concerning ultra-relativstic heavy ion collisions from RHIC, and it unambiguously demonstrates that there is sufficient energy density made for sufficient time to produce interesting forms of high energy density matter.

\section{Later Developments}

The ideas in Busza's and Bjorken's papers were both very well known and popular among Andrzej Bialas's group in Krakow. My own interest in the high energy limit of QCD arose from my attendance at a meeting of the Krakow School of Physics where both early experimental data from the SPS and data from HERA were shown together. Such a contemporaneous presentation was rare at that time, and led to thinking about how one might incorporate the effect of high gluon density into a hadron wave function[11],[12],[13], [14]. Once 
one understands that one is dealing with a high energy density of gluons in the initial state, one is led to a weak coupling description and ideas such as gluon saturation and the Color Glass Condensate. For nuclear collisions, this high energy density and highly coherent gluonic matter makes very strong color electric and magnetic fields, that strongly self interact[15],[16]. I think the basic elements needed for the description of high energy nuclear collisions includes the following elements

1. The Color Glass Condensate: Very high density of gluons that are the part of the hadron wave function that dominate high energy scattering processes.

2. The Strongly Interacting Quark Gluon Plasma: Matter produced in a high energy collision that behaves as a nearly perfect fluid.

\section{The Strongly Interacting Quark Gluon Plasma includes:}

- The Glasma: At early time, the matter is composed of highly coherent fields which are weakly coupled, but strongly interacting due to cohrence. Due to their strong interaction, this Glasma behaves as fluid. It eventually thermalizes, but this may take a long time relative to natural time scales.

- Thermalized Quark Gluon Plasma: When the matter is thermalized, it is not so coherent and presumably is a good fluid because of intrinsic strong to intermediate strength coupling

One of the goals of the RHIC, LHC and eRHIC programs is to test ideas concerning the various stages of hadronic collisions outlined above. The study of $p A$ collisions plays a particularly important role since it changes the balance between initial and final state interactions needed, and provides a way of disentangling them.

\section{Summary}

The subject of this meeting is largely about $p p, p A$ and $d A$ collisions. The results being discussed and the ideas for interpreting them have become richer and more important than anyone could have ever imagined when Busza wrote his review. The questions being asked are of fundamental importance for our understanding of strongly interacting matter, and they are simple in formulation, and answerable. The answers to many of these questions are either not yet known or generally accepted, and the conjectures are hotly disputed. As a clearer understanding develops through both experimental results and theoretical investigation, we hopefully will develop a consensus of understanding, and a picture that arises from a first principles understanding of QCD.

\section{Acknowledgements}

The research of L. McLerran is supported under DOE Contract No. DEAC02-98CH10886. 


\section{References}

[1] W. Busza, Acta Phys. Polon. B 8 (1977) 333.

[2] J. D. Bjorken, Lect. Notes Phys. 56 (1976) 93.

[3] A. Bialas, M. Bleszynski and W. Czyz, Nucl. Phys. B 111 (1976) 461.

[4] W. Busza, J. E. Elias, D. F. Jacobs, P. A. Swartz, C. C. Young and M. R. Sogard, Phys. Rev. Lett. 34, 836 (1975).

[5] R. Anishetty, P. Koehler and L. D. McLerran, Phys. Rev. D 22, 2793 (1980).

[6] J. D. Bjorken, Phys. Rev. D 27, 140 (1983).

[7] L. D. Landau and I. Pomeranchuk, Dokl. Akad. Nauk Ser. Fiz. 92, 535 (1953).

[8] A. B. Migdal, Phys. Rev. 103, 1811 (1956).

[9] A. Bzdak and V. Skokov, arXiv:1307.6168 [hep-ph].

[10] B. B. Back et al. [PHOBOS Collaboration], Phys. Rev. Lett. 85, 3100 (2000) [hep-ex/0007036].

[11] L. V. Gribov, E. M. Levin and M. G. Ryskin, Phys. Rept. 100, 1 (1983).

[12] A. H. Mueller and J. -w. Qiu, Nucl. Phys. B 268, 427 (1986).

[13] L. D. McLerran and R. Venugopalan, Phys. Rev. D 49, 2233 (1994) [hep$\mathrm{ph} / 9309289]$.

[14] L. D. McLerran and R. Venugopalan, Phys. Rev. D 49, 3352 (1994) [hep$\mathrm{ph} / 9311205]$.

[15] A. Kovner, L. D. McLerran and H. Weigert, Phys. Rev. D 52, 6231 (1995) [hep-ph/9502289].

[16] A. Kovner, L. D. McLerran and H. Weigert, Phys. Rev. D 52, 3809 (1995) [hep-ph/9505320]. 